\title{
The Noise Reduction over Wireless Channel Using Vector Quantization Compression and Filtering
}

\author{
Iman Elawady, Abdelmounaim Moulay Lakhdar, Khelifi Mustapha
}

Departement of Electronic, Tahri Mohammed University, Bechar, Algeria

\begin{tabular}{l} 
Article Info \\
\hline Article history: \\
Received Jun 29, 2015 \\
Revised Sep 7, 2015 \\
Accepted Oct 3, 2015 \\
\hline Keyword: \\
Codebook generation \\
Filtering \\
Gilbert-Elliot channel \\
Indices recovery \\
Vector quantization
\end{tabular}

\begin{abstract}
The transmission of compressed data over wireless channel conditions represents a big challenge. The idea of providing robust transmission gets a lot of attention in field of research. In this paper we study the effect of the noise over wireless channel. We use the model of Gilbert-Elliot to represent the wireless channel. The parameters of the model are selected to represent three cases of channel. As data for transmission we use images in gray level size $512 \times 512$. To minimize bandwidth usage we compressed the image with vector quantization also in this compression technique we study the effect of the codebook in the robustness of transmission so we use different algorithms to generate a codebook for the vector quantization finally we study the restoration efficiency of received image using filtering and indices recovery technique.
\end{abstract}

Copyright () 2016 Institute of Advanced Engineering and Science. All rights reserved.

\section{Corresponding Author:}

Iman Elawady,

Departement of Electronic,

Tahri Mohammed University,

B.P 417 BECHAR (08000), Algeria.

Email: imanelawady2@gmail.com

\section{INTRODUCTION}

The transmission of data over noisy channel make us concerned about the robustness of transmission and the effectiveness of restoration process in case of erroneous data. There is lot of parameters affect in the quality of received image. Some researchers think that the problem can be solved by optimize in the source coding as shown in the articles [1], [2] and [3], others think that the solution exists in the optimization in the channel coding as shown in the articles [4] and [5], other guesses think that we should make combination between the source and channel coding or what we call joint source channel coding (JSCC) as shown in the articles [6], [7] and [8], in this proposition they think we have to optimize the source and the channel coding according to the channel conditions which is unpredictable and depending on probability also it consumes lot of time and resources of processing unit because they are iterative algorithms.

In the optimization of the source coding they depend on adding some transforms to enhance the quality of reconstructed image but this is not a guarantee that the transmission is robust or that going to make the restoration process more efficient with less processing; however in the channel coding they create some algorithm that add some data as shown in the articles [9] and [10] (corrector bits or add some redundancy) in the original information or changing the data organization (interleaving) in way that we can estimate or guess the original information as shown in the article [11] or get it from the redundant data which is in general minimize the compression ratio and maximize in bandwidth usage.

The vector quantization provides high efficiency as a source coding technique with high compression ratio and reasonable quality; however we can't keep this enhancement after transmission. As shown in the article [12] that the indices are transmitted over a noisy channel, which is obviously most often the case, transmission errors usually occur (the received indices are not all the same of the transmitted 
indices). Since the vector quantization is a block coding technique the error also will be all the pixels in the block and to make restoration it becomes a big challenge because we don't have any information about the nature of pixels that contains in that block. Our proposition based on working on the nature of indices by using different codebooks and try to collecting some data that will be send and use in restoration process to study the influence of changing the codebook creation in the reception and restoration process and in the quality of received image.

This paper is organized as follows in the first part we try to introduce the concept of the vector quantization and the generation of indices that will be transmitted over the channel, after that the creation of codebook using two different approaches, than the parameters used for the model of Gilbert-Elliot as wireless channel. In third part we will introduce a technique of restoration (indices recovery). Finally we present the simulation results, discussion and conclusion.

\section{VECTOR QUANTIZATION}

Recently image compression, especially at low bit rate, has assumed a major role in applications such as storage on low memory devices, narrow-band channel transmitting, wireless transmitting and streaming data on the internet [13]. Shannon first suggested that encoding a sequence of samples from a source can provide better result than encoding individual samples in terms of compression efficiency [14].Image Data compression using vector quantization (VQ) has received a lot of attention.

Since VQ has a high coding efficiency and simple decoder architecture, it is very suitable for lowbit rate applications.

The general VQ algorithm has three main steps [15]:

- $\quad$ First divide the image into blocks (usually they are $2 \times 2,4 \times 4,8 \times 8$, or $16 \times 16$ ).

- After that a codebook with best estimation of blocks is constructed and indexed.

- Finally, the original image blocks are substituted by the index of best estimate code from the codebook.

The basic principle of vector quantization is to match each input vector with a code-vector in the codebook so that the distortion between the input vector and the chosen code-vector is minimum [14]. Quantization is an irreversible process so there is no way to find the original value from the quantized value [16]. The difference between the input and output signals of the quantizer generate the quantizing error, or quantizing noise [17].

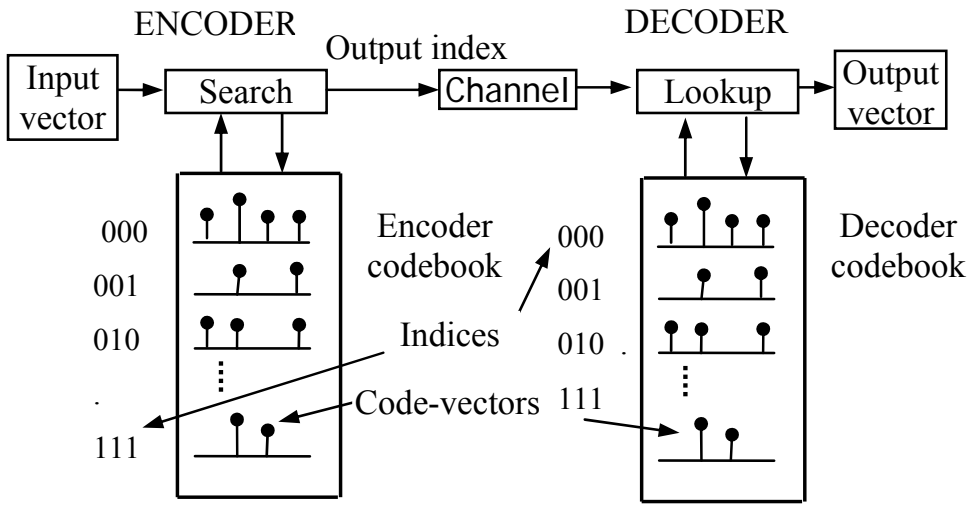

Figure 1. Vector quantization procedure

\section{GENERATION OF CODEBOOK}

The objective of codebook design is to minimize the combined reconstruction error over a representative training data set. If the training set is representative, the designed codebook will minimize the reconstruction error of input data however, except for the case of very small dimension. Codebook size, and/or training sets exhaustive search for the set of codebook vectors that minimized the total error is intractable problem [18]. A global codebook has one codebook for a class of images. The codebook is being derived from vectors of all images in the class. It is less overhead as compared to local codebook due to which it has lower performance. 


\subsection{Basic SOM Algorithm}

Kohonen proposed a Self-Organization MAP (SOM) for unsupervised neural net-work in 1980 [19]. Initially SOM is trained with the randomly initialized weight vectors and generate an initial codebook. The initial Codebook Generation algorithm is summarized as follows [20]:

Step 1: Transform the facial images in dataset to intensity variation vectors, and combine all data together into one training set.

Step 2: Specify the size of the codebook to $\mathrm{N}$ and initialize the codevectors by using continuous intensity variation vectors.

Step 3: Select a new training vector from the training set.

Step 4: Find the best matching codevector closest to the training vector.

Step 5: Move the best-matching and its neighborhood codevectors towards the training vector.

Step 6: Repeat from Step 3 until the map converges.

\subsection{Basic LBG Algorithm}

The design of optimal vector quantizers were proposed and extensively studied by Linde, Buzo, and Gray using a clustering approach, and is referred to as the LBG algorithm [21]. This algorithm is a generalization of the Lloyd-Max design algorithm for scalar quantization [22]. The LBG algorithm and other variations of this algorithm are based upon minimizing a distortion measure which represents the penalty or cost associated with the mapping. The LBG algorithm for codebook generation is as follows: [23]

Step 1. Given an arbitrary codebook, encode each input vector according to the nearest-neighbor criterion. Use a distance metric to compare all the input vectors to the encoded vectors, and then sum these errors (distances) to provide a distortion measure. If the distortion is enough small (less than a predefined threshold), then quit. If not, go to the step 2.

Step 2. For each codebook entry, compute the Euclidean centroid of all the input vectors encoded into that specific codebook vector.

Step 3: use the computed centroids as the new codebook, and go to step 1.

\section{TRANSMISSION CHANNEL (GILBERT-ELLIOTT)}

During the 1980s, the emergence of digital communication based on digital technologies promoted the second generation of mobile communication system and its standardization development, the application of digital technologies not only increased system capacity, but also made wireless business quality more reliable [24]. The Gilbert-Elliott model, often used for the modeling of a discrete channel with memory, is simply a Markov chain of two states: a state "GOOD" and "BAD" and that within each state, the channel behaves as a BSC of BER ' $\epsilon_{\mathrm{G}}$ ' in case of the state "GOOD" and ' $\epsilon_{\mathrm{B}}$ ' in case of the state "BAD".

We consider, in the following, three different channels that parameters are given in Table 4. Channel 1 present the more bad transmission conditions with an average duration of fading (channel in the state "BAD") four times greater than that of channels 2 and 3. It remains $80 \%$ of the time in the state "BAD". However, channel 3 remains only $20 \%$ of the time in this state. Note that the BER of the state "BAD" is $10 \%$ for the three channels considered and the mean BERs of these channels are respectively of the order of $8 \%$ for channel $1,5 \%$ for channel 2 and $2 \%$ for channel 3 .

Table 1. Parameters of the considered channels

\begin{tabular}{lccc}
\hline & Channel 1 & Channel 2 & Channel 3 \\
\hline$\epsilon_{\mathrm{G}}$ BER for the state "GOOD" & 0.001 & 0.001 & 0.001 \\
$\mathrm{C}_{\mathrm{B}}$ BER for the state "BAD" & 0.1 & 0.1 & 0.1 \\
$\mathrm{P}_{\mathrm{GB}}$ : Probability of transition from the "GOOD" to state "BAD" & 0.005 & 0.005 & 0.00125 \\
$\mathrm{P}_{\mathrm{BG}}$ : Probability of transition from the"BAD" to state "GOOD" & 0.00125 & 0.005 & 0.005 \\
$\mathrm{P}_{\mathrm{G}}$ : Probability that the channel is in state "GOOD" & 0.2 & 0.5 & 0.8 \\
$\mathrm{P}_{\mathrm{B}}$ : Probability that the channel is in state "bad" & 0.8 & 0.5 & 0.2 \\
mean BER & 0.0802 & 0.0505 & 0.0208 \\
Average length in bits of a fading (residence time in the state "BAD") & 800 & 200 & 200 \\
\hline
\end{tabular}

\section{INDICES RECOVERY}

This technique depends on collecting some verification information (bloc mean and complexity variation) is embedded into the indices of the VQ encoded data. First, we divide the indices matrix (code vectors) into sub blocs of size $\mathrm{w} \times \mathrm{W}$. for each indice we calculate the absolute difference between it vector mean value and the median mean value of neighboring code vector. After that, we keep the maximum absolute difference for each $\mathrm{w} \times \mathrm{w}$ sub bloc. 


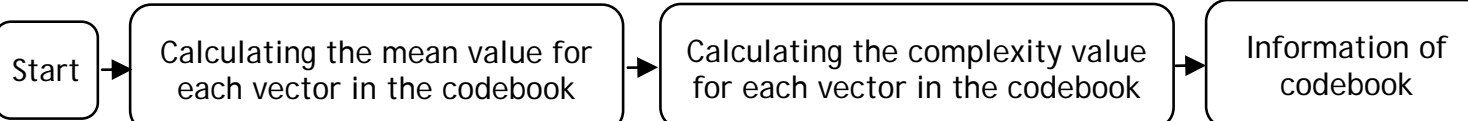

Figure 2. Verification information

In reception we have the received indices matrix that contains some erroneous indices. We calculate in reception the same information in emission. Then we compare it with the original information received which is the maximum for $\mathrm{w}^{\times} \mathrm{w}$ sub bloc. If the calculated value is higher than the max we replace it with the median value of neighboring indices.

\section{NOISE DETECTION}

Several sequences are generated during the impulse detection procedure. The first is a sequence of original indices matrix and its vector means, $x(i, j)$ and $\mathrm{Mx}(\mathrm{i}, \mathrm{j})$ respectively. $(\mathrm{i}, \mathrm{j})$ is position of indice, it can be $1 \leq \mathrm{i} \leq \mathrm{M}, 1 \leq \mathrm{j} \leq \mathrm{N}$ where $\mathrm{M}$ and $\mathrm{N}$ are the number of the indices in horizontal and vertical direction respectively, The second is a variation matrix, $f(\mathrm{Mx}(\mathrm{i}, \mathrm{j}))$ is used to indicate whether the indice at $(\mathrm{i}, \mathrm{j})$ in noisy indices detected as noisy or noise free. The third is a sequence of received indices matrix and its means, $x^{\prime}(i, j)$ and $M x^{\prime}(i, j)$ respectively [9].

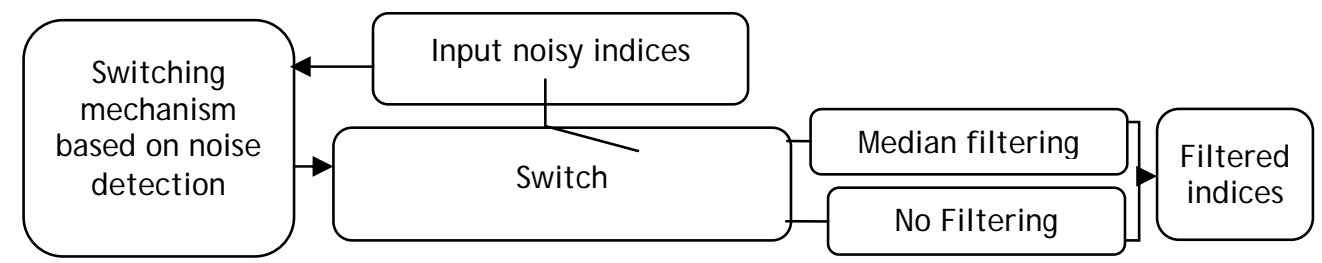

Figure 3. Block diagram of proposed Filter

1. Lets take a $(2 p+1) \times(2 p+1)$ window around $M x(i, j)$ means $M x(i+k, j+l)$ where $-p \leq(k, 1) \leq p$, and $p \geq 1$. Where

$$
M x(i, j)=\frac{1}{q \times q} \sum_{k=1}^{q \times q} x_{k}(i, j)
$$

$\mathrm{x}_{\mathrm{k}}$ is the gray level of code vector pixel. $\mathrm{q} \times \mathrm{q}$ is the bloc size of quantified pixels.

2. Find Median value of this window $m(i, j)$

$$
\mathrm{m}(\mathrm{i}, \mathrm{j})=\operatorname{Median}[\mathrm{Mx}(\mathrm{i}+\mathrm{k}, \mathrm{j}+\mathrm{l})]
$$

3. Find absolute difference between $\mathrm{Mx}(\mathrm{i}, \mathrm{j})$ and $\mathrm{m}(\mathrm{i}, \mathrm{j})$, and assign

$$
f(M x(i, j))=|x(i, j)-m(i, j)|
$$

4. Find the maximum value of $f$ for $1 \leq(i, j) \leq w$

5. Send just this maximum values with original indices matrix, $x(i, j)$. we have $N V=M \times N / w \times w$ values. This is negligible.

6. in the receiver, Calculate

$$
f\left(x^{\prime}(i, j)\right)=\left|x^{\prime}(i, j)-m^{\prime}(i, j)\right|
$$

With :

$$
m^{\prime}(\mathrm{i}, \mathrm{j})=\operatorname{Median}\left[\mathrm{Mx}^{\prime}(\mathrm{i}+\mathrm{k}, \mathrm{j}+\mathrm{l})\right]
$$


7. If $f\left(M^{\prime}(i, j)\right)>\max [f(M x(i, j))] x^{\prime}(i, j)$ is detected as noisy then the estimated value of $x^{\prime}(i, j)$ will be modified as

$$
\hat{x}(i, j)=\left\{\begin{array}{c}
m^{\prime}(i, j) \text { if } x^{\prime}(i, j) \text { is erroneous } \\
x^{\prime}(i, j) \text { otherwise }
\end{array}\right.
$$

After all that we make the same algorithm but we replace the original indices matrix mean $\mathrm{Mx}(\mathrm{i}, \mathrm{j})$ by original indices matrix complexity $\mathrm{Cx}(\mathrm{i}, \mathrm{j})$.

$$
\operatorname{Cx}(\mathrm{i}, \mathrm{j})=\operatorname{maxk}(\mathrm{xk}(\mathrm{i}, \mathrm{j}))-\operatorname{mink}(\mathrm{xk}(\mathrm{i}, \mathrm{j})) \mathrm{k}=1, . ., \mathrm{qxq}
$$

\section{RESULTS AND ANALYSIS}

For the simulation we use four images (lena, Boat, Goldhill, Peppers) size 512x512, in the gray level, the block size is $4 \times 4$, and universal codebook size is $256 \times 16$, the quality of reconstructed image without transmission using LBG codebook and SOM codebook is represented as shown below:

Table 2. The quality of reconstructed image $(\mathrm{dB})$

\begin{tabular}{ccc}
\hline & LBG codebook & SOM codebook \\
\hline lena & 30.3848 & 29.7034 \\
Boat & 28.9948 & 28.0961 \\
Goldhill & 29.4160 & 29.0325 \\
Peppers & 25.8798 & 25.1396 \\
\hline
\end{tabular}

For transmission over wireless channel the parameters of Gilbert-Elliott model are selected as indicated in table 1 . The simulation results present: received image, filtered, recovered indices, and recovered indices with filtering.
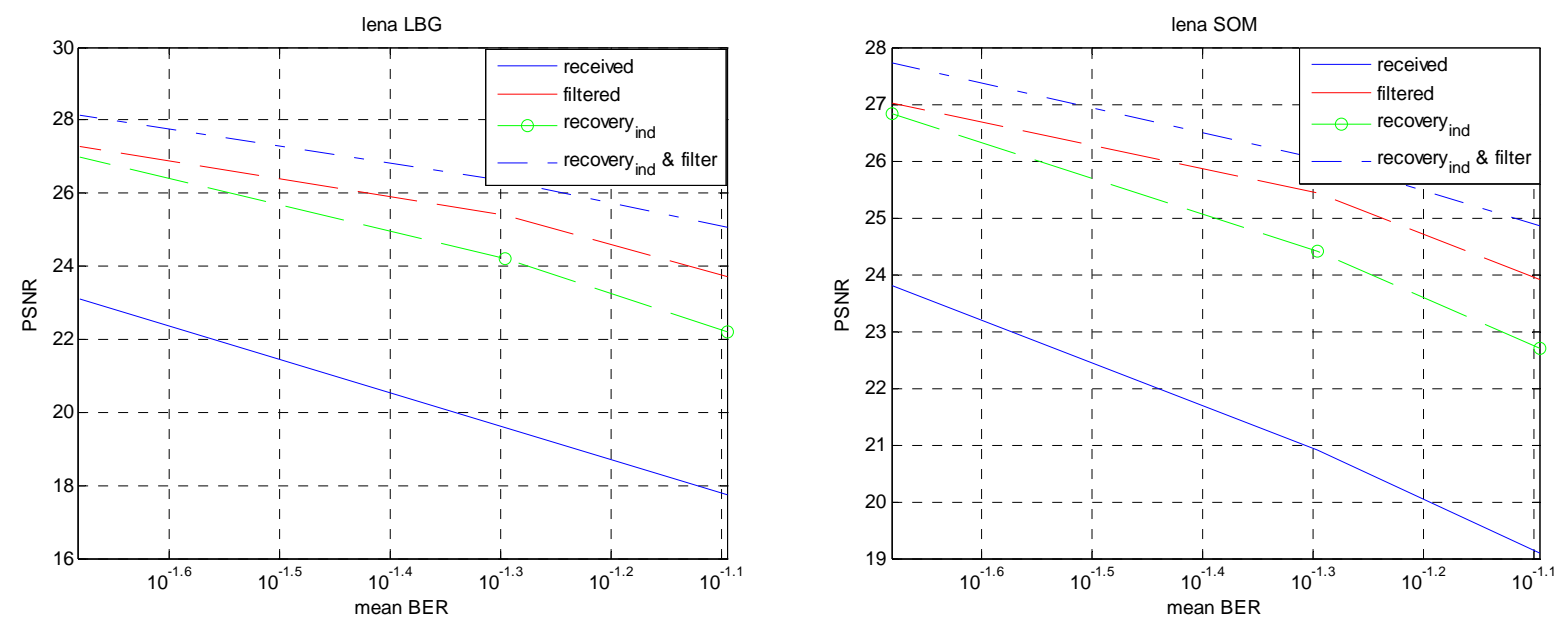

Figure 4. Received and restored image (lena) (left with: LBG codebook. Right with: SOM codebook) 

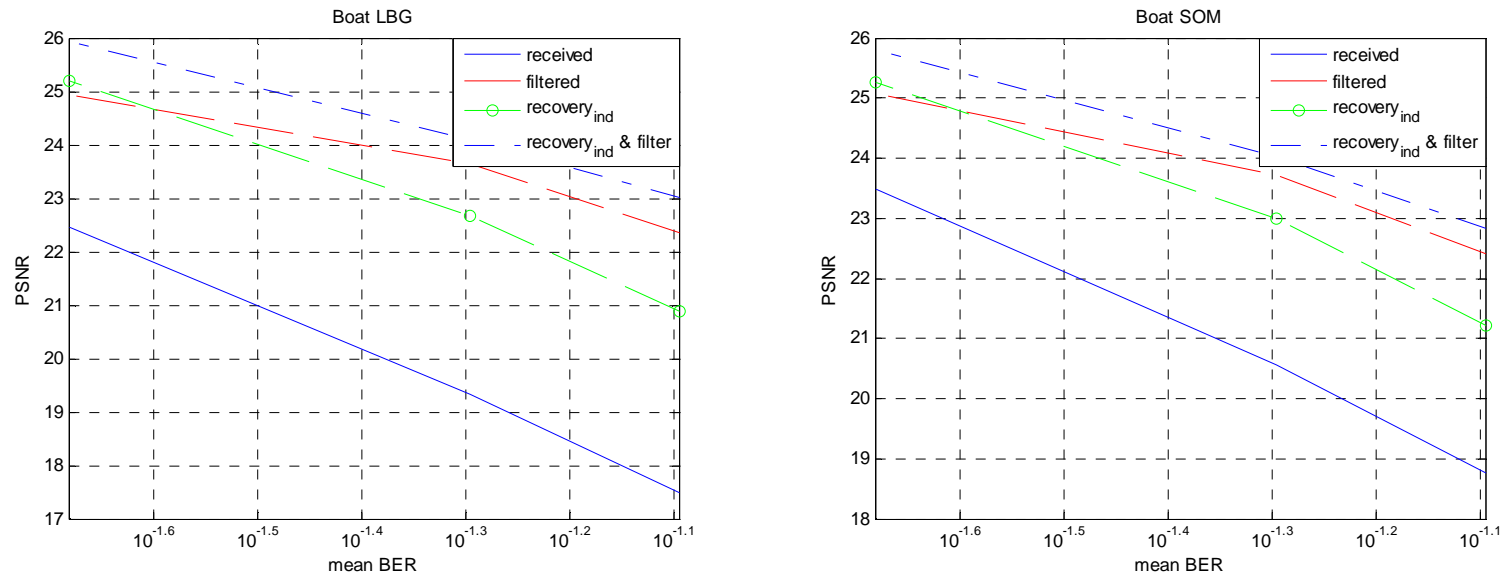

Figure 5. Received and restored image (Boat) (left with: LBG codebook. Right with: SOM codebook)
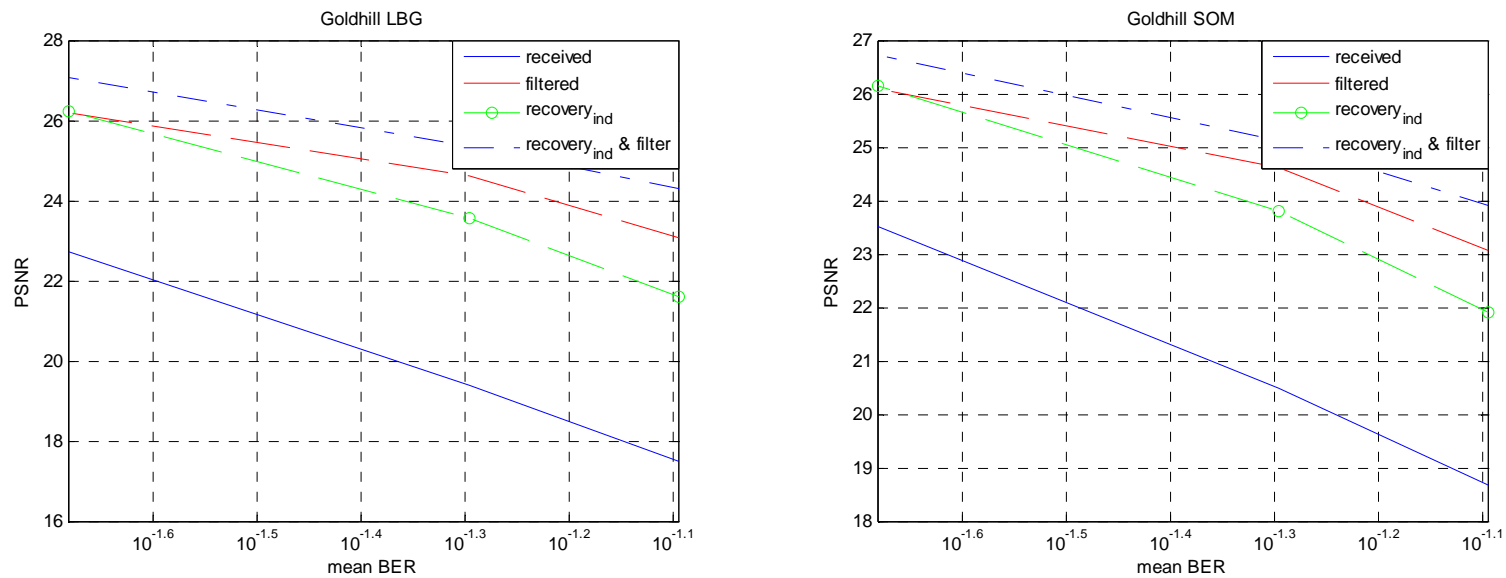

Figure 6. Received and restored image (Goldhill) (left with: LBG codebook. Right with: SOM codebook)
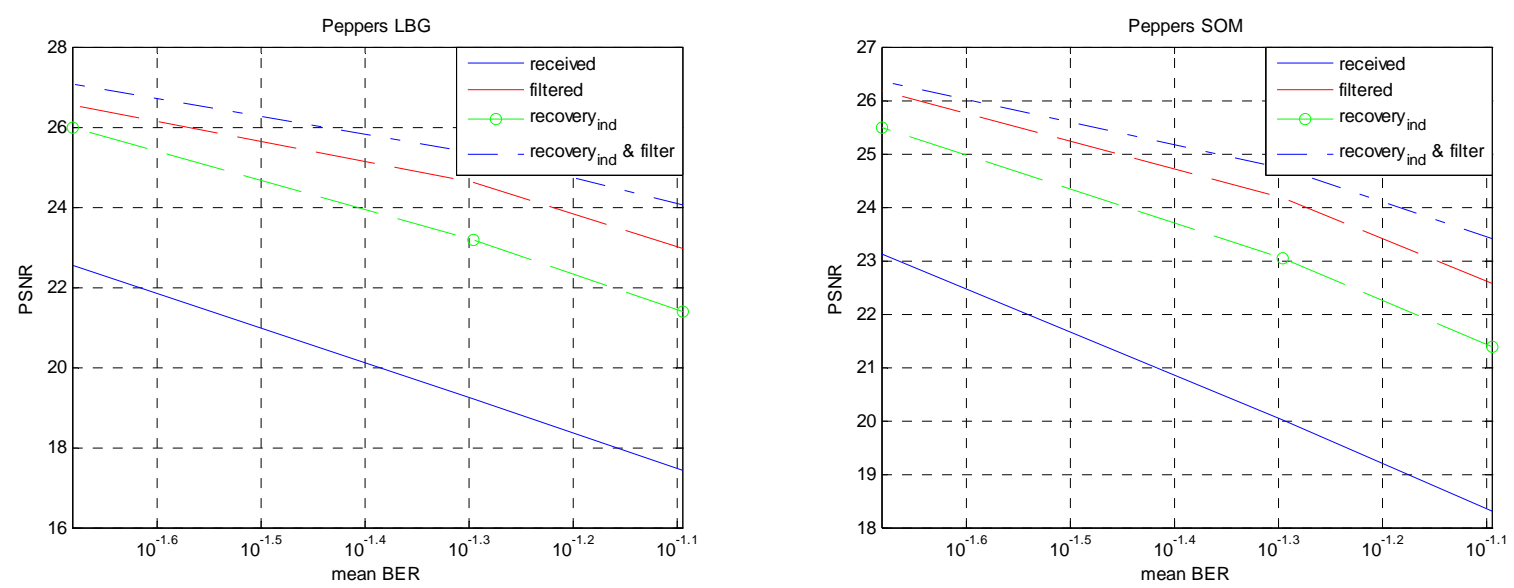

Figure 7. Received and restored image (Peppers) (left with: LBG codebook. Right with: SOM codebook)

Before we comment in these results we should see the backgrounds of each technique for codebook creation and the relationship between code vectors in the codeword. 
As mentioned in [25] and [26] the main difference between LBG and SOM algorithms is in the order of the codevector indexes. The LBG algorithm does not define any order in the codebook; the codewords for the model vector can be selected arbitrarily. On the other hand the codebook trained using the SOM algorithm has an internal order, adjacent codebook entries. This is due to the fact that SOMs use the method of competitive learning [27]. For more details the articles [28] and [29] provide more information about differences in codebook creation between LBG and SOM algorithms.

That's why the simulation results show that SOM provid better results for received image because even we get an error in indices the erroneous index will be close to the original one.

After the restoration process the combination between indices recovery and standard median filter provide the best result however we can't benefit on this improvement achieved in received image using SOM codebook for restoration process. The collected information using LBG codebook for indices recovery will be with high precision because there is big difference between codevectors so all collected information can specify the right block for recovery process however in SOM codebook the collected information can't give us or detect a specific block in the recovery process.

Also we shouldn't forget that the quality of reconstructed image using LBG is better than SOM. There are many articles propose a solution as shown in [30] we can enhance the quality of image by using Savitzky-Golay polynomial; in article [31] the enhancement can be achieved by using optimal Kohonen topological map to determine the optimal codebook and also avoid the problem of "dead units" that can arise for example with the LBG algorithm, another suggestion to solve this problem is mentioned in articles [32] and [33] by applying hierarchical SOM.

The simulation results prove that we can enhance the quality of received image using SOM codebook also provide an efficient restoration technique using noise reduction and median filter. Our suggestion look simple and easy compared with other suggestions which make us interested to make lot of research based on proposed solution.

\section{CONCLUSION}

In this paper we provide a suggestion that can improve the quality of received image without minimize the compression ratio or maximize the bandwidth usage as shown in [28] and [29]. Our suggestion depending on the relationship between produced indices in the codevectors. We also test the efficiency of restoration in this case to benefit from the maximum improvement achieved. The use of SOM codebook provides good quality of received image compared with LBG codebook. We also discovered that the combination between indices recovery and standard median filter provide the best results. However, we still have a problem in restoration process to specify the collected data in SOM codebook. The solution can be by using methods shown in [30], [31], [32] and [33] or by using transforms that can analyze the image to improve the quality of reconstructed image and also help us to collect information with more precision.

\section{REFERENCES}

[1] Jun Chen,Toby Berger .Robust Distributed Source Coding. IEEE Transactions on Information Theory. August 2008; 54(8); 3385-3398.

[2] S.K. Muttoo, Sushil Kumar .Robust Source Coding Steganographic Technique Using Wavelet Transforms. International Journal of Information Technology Bharati Vidyapeeth's Institute of Computer Applications and Management (BVICAM). New Delhi, December, 2009; 1(2); 91-96.

[3] Michelle Effros. Robustness to Channel Variation in Source Coding for Transmission Across Noisy Channels. IEEE International Conference on Acoustics, Speech, and Signal Processing. 21-24 Apr 1997; 4; 2961-2964.

[4] Mannava Srinivasa Rao, Boppana Swati Lakshmi, Dr.Panakala Rajesh Kumar. Wireless Image Transmission over Noisy Channels Using Turbo Codes and De-noising Filters. International Journal of Engineering Research and Applications. September- October 2012; 2(5); 1936-1941.

[5] Laura Toni, Yee Sin Chan, Pamela C. Cosman, Laurence B. Milstein .Channel Coding for Progressive Images in a 2-D Time-Frequency OFDM Block With Channel Estimation Errors. IEEE Transactions on Image Processing. November 2009; 18(11); 2476-2490.

[6] Wei Xiang, Steven S. Pietrobon and S. Adrian Barbulescu .Iterative Source-Channel Decoding for Robust Image Transmission. Australian Communication Theory Workshop Proceedings. 2003; 05-07.

[7] Ch. Rama Krishna Reddy, M.Padmaja, Dr. K.Sri Rama Krishna, K.Prasuna .Image Transmission Using Adaptive Channel Coding for Electronic Toll Collection System. International Journal of Electronics \& Communication Technology (IJECT). June 2011; 2 (2); 60-65.

[8] Jianfei Cai, Chang Wen Chen. Robust Joint Source-Channel Coding for Image Transmission Over Wireless Channels. IEEE Transactions on Circuits and Systems for Video Technology. September 2000; 10 (6); 962-966.

[9] A. Moulay Lakhdar, M. Beladgham, M.Anane, Ben Aissa. M N oise reduction of VQ Encoded Medical. Computing and Control Applications (CCCA) IEEE Conference. Tunisia March 2011; 1-6.

The Noise Reduction over Wireless Channel Using Vector Quantization Compression and ...(Iman Elawady) 
[10] Usama S. Mohammed, H.A. Hamada. Image transmission over OFDM channel with rate allocation scheme and minimum peak to average power ratio. Journal of Telecommunications. May 2010; 2(2); 70-78.

[11] Cornelius Hellge, Valentina Pullano, Manuel Hensel, Giovanni E. Corazza, Thomas Schierl, Thomas Wiegand. Mobile TV with long Time Interleaving and Fast Zapping. IEEE International Conference on Multimedia and Expo Workshops. 2012.

[12] Abdelmounaim Moulay Lakhdar, Mustapha Khelifi, Mohammed Beladgham, Mohammed Ben Aissa,Abdesselam Bassou. Image Vector Quantization Codec Indexes Filtering. Serbian Journal of Electrical Engineering. June 2012; $9(2) ; 263-277$.

[13] Xiaofeng Wu, Shigang Hu, Zhiming Li, Zhijun Tang, Jin Li, Jin Zhao. Comparisons of Threshold EZW and SPIHT Wavelets Based Image Compression Methods. TELKOMNIKA Indonesian Journal of Electrical Engineering. March 2014; 12 (3); 1895-1905.

[14] Ajol Kumar Ray,Tinku Acharya.Information Technology Principles and Applications. Prentic Hall of India,2004

[15] N.M. Nasrabadi, R.A. King. Image coding using vector quantization: a review. IEEE Transactions on Communications. Aug. 1988; 36 (8); 957-971.

[16] Yun Q. Shi, Huifang Sun. Image and Video Compression for Multimedia Engineering. CRC Press, second edition, 2008.

[17] Zhou Wang, Alan C.Bovik. Mean squared error love it or leave it. IEEE signal processing magazine. January. 2009; 99-117.

[18] Ronald G. Driggers. Encyclopedia of Optical Engineering. Sep 1, 2003; 2(3).

[19] Cheng-fa Tsai, Yu-Jiun Lin. Advances in Image and Video Technology. Pacific Rim Symposium on Image and Video Technology (PSIVT). Tokyo, Japan January 13-16, 2009.

[20] Qiu Chen, Koji Kotani, Feifei Lee, Tadahiro Ohmi. A Codebook Design Method for Robust VQ-Based Face Recognition Algorithm. J. Software Engineering \& Applications, 2010; 3(2); 119-124.

[21] Yoseph L, Andres B, Robert M.G. An algorithm for vector quantizer design. IEEE Trans, on Communications .January $1980 ; 28(1) ; 84-95$.

[22] Al Bovik. Handbook of Image and Video Processing. Academic Press, 2000.

[23] Scott E Umbaugh. Computer Imaging: Digital Image Analysis and Processing . CRC Press. Jan 27, 2005.

[24] Jianbin Xue, Songbai Li.Transmission Performance Research of Digital Modulation Signals in AWGN Channel. TELKOMNIKA Indonesian Journal of Electrical Engineering. February 2013; 11(2); 991-997

[25] Abdelkader Amraoui, Pierre Dufour. Robust Vector Quantization for Noisy Channels Using a Self-Organizing Pseudo-Map. Robust Speech Recognition for Unknown Communication Channels Pont-a-Mousson, France April 17-18, 1997; 135-138.

[26] M. Ettaouil, Y. Ghanou, K. El Moutaouakil, M. Lazaar.Image Medical Compression by A new Architecture Optimization Model for the Kohonen Networks. International Journal of Computer Theory and Engineering. April $2011 ; 3(2) ; 204: 210$.

[27] Norbert Ádám. A Speech Analysis System Based on Vector Quantization Using the LBG Algorithm and SelfOrganizing Maps. International Journal of Computer and Information Technology. September 2014 ; 03 (05); $952-$ 957.

[28] Ashraf R, Akbar M. Adaptive Architecture Neural Nets for Medical Image Compression. IEEE International Conference on Engineering of Intelligent. Systems, 2006; 1-4.

[29] Xu, Lixin, Liu, W.Q., Venkatesh, Svetha. A two stage vector quantization approach via self-organizing map. in ICSP' $02:$ 6th International Conference on Signal Processing proceedings IEEE Piscataway N. J. 2002;1;913-916.

[30] Tomi Kinnunen, Teemu Kilpeläinen, Pasi Fränti. Comparison of Clustering Algorithms in Speaker Identification. Pattern Recognition Letters archive. Elsevier Science Inc. New York, NY, USA .October 2011; 32(13); 1604-1617.

[31] Mohamed Ettaouil,Mohamed Lazaar. Compression of Medical Images using Improved Kohonen Algorithm. Special Issue of International Journal of Computer Applications (0975 - 8887) on Software Engineering. September 2012; 4 (6);111-117.

[32] G Boopathi, Dr. S. Arockiasamy. An Image Compression Approach using Wavelet Transform and Modified Self Organizing Map. IJCSI International Journal of Computer Science Issues. September 2011; 8 (5); 1694-0814.

[33] Cheng-Fa Tsai, Chen-An Jhuang, Chih-Wei Liu.Gray Image Compression Using New Hierarchical SelfOrganizing Map Technique. The 3rd Intetnational Conference on Innovative Computing Information and Control (ICICIC'08) IEEE, June 2008; 544.

\section{BIOGRAPHIES OF AUTHORS}

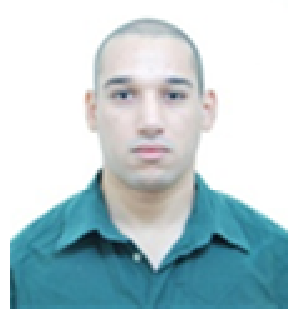

Iman Elawady was born in Bechar, Algeria. He received the dipl. License from the University of Bechar, Algeria, in 2009 and the Master degree in digital communication systems from University of Bechar, Algeria. in 2011. Actuelly, he prepare the doctoral degree at University of Bechar, Algeria. His main interests are Image processing transmission, compression, electromagnetic field and antenna array. Correspondence address: Bechar University, Department of Electronic, Bechar, Algeria,

Email: imanelawady2@gmail.com

IJECE Vol. 6, No. 1, February $2016: 130-138$ 


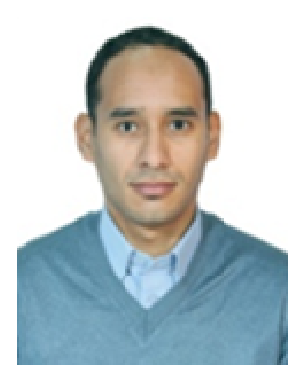

A. Moulay Lakhdar got the Engineering Degree in Telecommunication in 2000 at the Institute of Telecommunications in Oran. Magister was the second degree in Signal and telecom at Djillali LIABES university of Sidi Bel Abbes in 2003. From 2004 up present, he worked in the Bechar University as lecturer. Since May 2009, he graduated PhD Es Sciences at Sidi Bel Abbes. He do his research activity at the Bechar University and Communications, Architecture and Media Laboratory (CAMR) (Djillali Liabes University). His research interests are Image transmission, Image processing, and digital transmission performances. Correspondence address: Bechar University, Department of Electronic, Bechar, Algeria,

Email: moulaylakhdar78@yahoo.fr

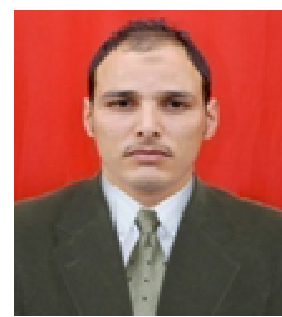

Khelifi Mustapha was born in Ain sefra, Naama. He received the degree in electrical engineering from the University DR Moulay Tahar Saida, Algeria, in 2009 and the Master degree in signal and digital communication from University of Bechar, Algeria.in 2011. Actuelly, he prepares the doctoral degree at University of Bechar, Algeria. His main interests are Image processing, compression, channel and source coding. Correspondence address: Bechar University, Department of Electronic, Bechar, Algeria,

Email: Khelifi_m@yahoo.fr 